\title{
Refuge
}

Canada's Journal on Refugees

revue canadienne sur les réfugiés

\section{Patrouille des frontières nationales et représentations racialisées: Analyse de commentaires en ligne sur les réfugiés syriens au Québec}

\section{Mathieu Forcier}

Volume 35, Number 1, 2019

Racialized Refuge

URI: https://id.erudit.org/iderudit/1060674ar

DOI: https://doi.org/10.7202/1060674ar

See table of contents

Publisher(s)

Centre for Refugee Studies, York University

ISSN

0229-5113 (print)

1920-7336 (digital)

Explore this journal

Cite this article

Forcier, M. (2019). Patrouille des frontières nationales et représentations racialisées: Analyse de commentaires en ligne sur les réfugiés syriens au Québec. Refuge, 35(1), 43-60. https://doi.org/10.7202/1060674ar

\section{Article abstract}

This article analyzes comments published on the Facebook pages of major Quebec media outlets about the Canadian resettlement plan for Syrian refugees in 2015. The study seeks to probe the particular configuration of the normalization of white ethnic majorities' national belonging under the mode of worry. Five commonplace ideas are identified in the negative discursive framing of the arrival of Syrian refugees: numbers, financial burden, national responsibility, culture, and security. The results show that racialized exclusion can operate without recourse to openly racist language, notably through the representation of the "people" as a victim of multicultural elites.
Copyright (c) Refuge: Canada's Journal on Refugees, 2019

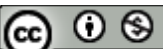

This document is protected by copyright law. Use of the services of Érudit (including reproduction) is subject to its terms and conditions, which can be viewed online.

https://apropos.erudit.org/en/users/policy-on-use/ 


\title{
Patrouille des frontières nationales et
} représentations racialisées:

\section{Analyse de commentaires en ligne sur les réfugiés syriens au Québec}

\author{
MATHIEU FORCIER
}

\section{Résumé}

Larticle analyse des commentaires publiés sur les pages de grands médias québécois sur Facebook en lien avec le plan canadien de réinstallation de réfugiés syriens en 2015. Létude vise à sonder la configuration particulière de la normalisation de lappartenance nationale des majorités ethniques blanches sous le mode de l'inquiétude. Cinq lieux communs associés au déni du racisme et à la présentation positive de soi sont identifiés dans le cadrage discursif négatif de l'arrivée des réfugiés syriens, soit le nombre, le fardeau financier, la responsabilité nationale, la culture et la sécurité. Les résultats montrent que l'exclusion racialisée peut être opérée sans recourir à un langage ouvertement raciste, notamment à travers la représentation du "peuple» comme victime des élites multiculturalistes.

\begin{abstract}
This article analyzes comments published on the Facebook pages of major Quebec media outlets about the Canadian resettlement plan for Syrian refugees in 2015. The study seeks to probe the particular configuration of the normalization of white ethnic majorities' national belonging under the mode of worry. Five commonplace ideas are identified in the negative discursive framing of the arrival of Syrian refugees: numbers, financial burden, national responsibility, culture, and security. The results show that racialized exclusion can operate without recourse to openly racist language, notably through the representation of the "people" as a victim of multicultural elites.
\end{abstract}

(C) Mathieu Forcier, 2019. This open-access work is licensed under a Creative Commons Attribution-NonCommercial 4.0 International Licence, which permits use, reproduction, and distribution in any medium for non-commercial purposes, provided the original authorship is credited and the original publication in Refuge: Canada's Journal on Refugees is cited.
Cette œuvre en libre accès fait l'objet d'une licence Creative Commons Attribution-NonCommercial 4.0 International License, laquelle autorise l'utilisation, la reproduction et la distribution de l'œuvre sur tout support à des fins non commerciales, pourvu que l'auteur ou les auteurs originaux soient mentionnés et que la publication originale dans Refuge: revue canadienne sur les réfugiés soit citée. 


\section{Introduction}

u moment où les images du jeune Alan Kurdi font la une des journaux, le Canada est en pleine campagne 1 électorale et cet événement s'impose comme enjeu clé ${ }^{1}$. Le parti libéral remporte les élections avec la promesse d'accueillir 25 ooo réfugiés d'ici le ier janvier $2016^{2}$. L’opinion publique est alors fortement polarisée ${ }^{3}$. Au Québec, des regroupements anti et pro-réfugiés se forment, des banderoles sont déployées sur la place publique et, au lendemain des attentats de Paris, une pétition intitulée «non à l'immigration de réfugiés syriens» récolte 50000 signatures en deux jours. 60\% des Québécois s'opposent alors au projet du gouvernement Trudeau et un sur cinq affirme vouloir fermer la porte à ces réfugiés ${ }^{4}$. Les citoyens sont aussi particulièrement vocaux sur les médias sociaux. Il importe d'y porter attention de façon à mieux comprendre les dynamiques de racialisation du refuge au Canada et au Québec de façon plus spécifique. Ces médias sont un espace fructueux pour le racisme et les stratégies de racialisation risquent de différer de celles rencontrées hors-ligne. Ces dernières renvoient au processus de catégorisation sociale qui construit et naturalise l'Autre comme porteur de différences insurmontables ${ }^{5}$.

Précisons que les compétences en matière d'immigration sont partagées entre les gouvernements canadien et québécois ${ }^{6}$. On observe au Québec une croissance de la part de personnes qui estiment qu'il y a «trop d'immigrants ${ }^{7}$. En plus de la discrimination systémique, notamment sur le marché du travail, au niveau du discours et des perceptions, la société québécoise connaît une banalisation de l'islamophobie, notamment à travers une multitude de débats sur la laïcité ${ }^{8}$. La problématisation sociale des musulmans est aussi visible dans différentes études et sondages d'opinion?

Lobjectif est ici de sonder la normalisation de la représentation du territoire national comme devant être protégé face aux forces de la globalisation et en particulier les mobilités jugées «à risque». La présente conjoncture est caractérisée par la "droitisation» de la politique où l'Autre racialisé est soumis au regard suspicieux des acteurs institutionnels et des membres de la société civile du fait de sa représentation comme source potentielle de problèmes, comme porteur de menaces culturelles, économiques ou sécuritaires. Ce climat de suspicion est tel que les discours sécuritaires, assimilationnistes et restrictifs à légard de l'immigration se sont normalisés au sein des partis mainstream ${ }^{10}$.

Lassociation entre migrations et insécurité est désormais un thème qui appartient au courant dominant, ce qui est d'autant plus clair depuis le Brexit et lélection de Trump. Les deux campagnes ont misé sur la peur de l' «immigration de masse», l'idée de reprise du contrôle des frontières et le ressentiment du peuple, des citoyens ordinaires (blancs) oubliés par les élites pro-globalisation et inquiets d'une perte relative de privilèges ${ }^{11}$. Il s'agit alors de mettre en lumière les articulations entre nationalisme et racialisation dans le discours de citoyens s'exprimant dans l'espace public numérique. Les analyses critiques du discours demeurent encore principalement centrées sur les élites, que ce soit la classe politique ou les médias, ce qui tend à négliger le rôle toujours actif des citoyens " ordinaires $»^{12}$, aujourd'hui plus que jamais perceptible par la culture du commentaire en ligne. Suivant notre cadre analytique inspiré des théories critiques du nationalisme, de la race et de la blanchité ${ }^{13}$, les médias sociaux sont vus comme de nouveaux sites de pouvoir où le discours est influencé par les inégalités racialisées et participe aussi à leur reproduction ${ }^{14}$. Comme les discours top-down généralement étudiés, ceux qui sont construits par le bas peuvent aussi favoriser la stigmatisation et la discrimination.

\section{Nationalisme et racisme sur Internet}

Internet offre de nouveaux moyens d'expression de la xénophobie et du racisme, exacerbant la discrimination de catégories sociales déjà marginalisées ${ }^{15}$. La littérature analysant les discours nationalistes et racistes en ligne est encore relativement récente et les études canadiennes sont d'ailleurs peu nombreuses ${ }^{16}$. La recherche s'est beaucoup penchée sur le recours au web par les suprématistes blancs et les partis d'extrême-droite ${ }^{17}$. Or, le focus sur les formes de racisme ${ }^{18}$ ouvert tend à perdre de vue les formes plus subtiles et plus en phase avec la norme culturelle dans l'espace public ${ }^{19}$. Les médias sociaux et les sections commentaires ont fait exploser la visibilité des attitudes xénophobes et racistes, ouvrant ainsi un nouveau terrain de recherche ${ }^{20}$. Globalement, la littérature montre qu'Internet est un espace où cohabitent des formes ouvertes et haineuses de racisme et des formes plus subtiles et socialement acceptables, notamment à travers les représentations de l'immigration comme menace pour l'identité nationale ${ }^{21}$. Si les discours à tendances xénophobes et racistes sont davantage visibles et décomplexés en ligne, leur prévalence serait moindre sur des plateformes qui demandent aux utilisateurs de s'identifier publiquement ${ }^{22}$.

Plusieurs études démontrent que les individus ont recours au déni du racisme et des privilèges liés à la blanchité dans les discussions en ligne tout en mettant de l'avant des postures exclusivistes ou discriminatoires ${ }^{23}$. Des membres du groupe majoritaire peuvent éviter les accusations de racisme en s'en prenant au système ou aux élites plutôt qu'aux minorités de façon directe ${ }^{24}$. De plus, les musulmans sont régulièrement les cibles privilégiées et les discours ne se limitent pas au langage haineux. Des représentations empreintes de peur et de suspicion construisent un Autre musulman mettant à risque la culture et les valeurs nationales, la cohésion sociale, l'égalité entre les hommes et les femmes, la sécurité nationale etc. ${ }^{25}$. Davantage de recherches sont nécessaires pour 
mieux suivre les nouveaux modes d'expression des opinions populaires. Comme le note Gavan Titley, «online communications and networked social media interactions provide key sites for the delineation of hierarchies of belonging, and the expansive rehearsal and contestation of racializing discourses, tropes and rationalities ${ }^{26}$.

Cet article présente une analyse critique de discours sur les réfugiés syriens à travers les commentaires de lecteurs de médias québécois publiés sur Facebook. Il s'inscrit dans le cadre d'un projet de recherche sociologique dont l'objectif est de jeter un éclairage nouveau sur la stigmatisation de certaines catégories d'immigrants ${ }^{27}$ et certaines minorités racialisées. Les représentations racialisées de ces populations portent atteinte à la dignité de leurs membres, à leur reconnaissance comme membres égaux de la société et les déshumanisent en les réduisant à des catégories identitaires essentialisées légitimant des pratiques d'exclusion et de subordination sociale ${ }^{28}$. Comme le soulignent bien Hughey et Daniels, "given that the Internet is becoming the major means for the production of public opinion and the dominant consensus on ethnic and racial affairs, this matter is more than academic $»^{29}$. Lanalyse des discours sur la «nation» et ses Autres est nécessaire pour déstabiliser la reproduction de la domination sociale des majoritaires sur les minorités racialisées ${ }^{30}$.

\section{Repères théoriques}

La nation est ici conceptualisée comme une catégorie discursive héritée de la modernité coloniale et dont les formes d'articulation avec l'ethnicité et la race varient historiquement ${ }^{31}$. Le concept de "patrouille des frontières ${ }^{32}$ est adopté pour désigner l'utilisation de marqueurs symboliques servant à décider qui est inclus/exclus des frontières ${ }^{33}$ de la communauté nationale en fixant les limites de l'incorporation de la différence. Suivant Stuart Hall, le discours est entendu comme un mode de représentation qui informe les perceptions et les pratiques et à travers lequel le pouvoir opère ${ }^{34}$. Lanalyse suit son approche conjoncturelle en situant les discours dans la conjoncture contemporaine marquée par la globalisation néolibérale, la croissance des inégalités, les mouvements migratoires Sud-Nord et le rejet du multiculturalisme ${ }^{35}$. Dans un contexte où les sociétés occidentales sont représentées de façon hégémonique comme étant post-raciales, l'analyse de la racialisation doit être attentive aux mécanismes plus ou moins subtils ${ }^{36}$. Le recours aux arguments culturalistes et à la victimisation des «gens ordinaires» (blancs) est aujourd'hui central pour à la fois nier le racisme et reproduire des identités nationales racialisées ${ }^{37}$. Depuis le tournant du millénaire, ces débats focalisent particulièrement sur l'idée racialisée du musulman comme «Autre ingouvernable» ${ }^{38}$.
Nous analysons les dynamiques d'inclusion différentielle et sélective et la différenciation entre la «bonne» et la «mauvaise» diversités ${ }^{39}$. On entend par là l'inclusion d'une diversité perçue comme un atout pour la nation et l'exclusion concomitante d'une diversité racialisée comme étant culturellement incompatible, pesant sur les finances publiques et posant un risque pour la sécurité nationale ${ }^{40}$. Lanxiété, la peur et l'insécurité médiatisent de plus en plus l'inclusion différenciée, la société étant vue comme devant être défendue et sécurisée, ce qui implique de réguler des formes de diversité dites menaçantes ${ }^{41}$.

Suivant Ghassan Hage, les débats sur l'immigration et l'identité correspondent à des rituels nationalistes caractérisés par la culture de l'inquiétude, désormais la forme normale de l'expression d'appartenance nationale ${ }^{42}$. À travers ces pratiques discursives, les membres de la majorité ethnique blanche peuvent réaffirmer leur droit dêtre inquiets pour leur nation et de fixer les limites de l'inclusion au sein de leur chez soi (home) en s'exprimant sur le mode de l'«appartenance gouvernementale». Plusieurs études québécoises montrent que les récents débats sociaux sur la «diversité» ont été largement dominés par la majorité ethnique, que les voix de membres des minorités ciblées - en particulier les femmes musulmanes - ont été, sinon discréditées, peu entendues et que ces débats ont servi à la reproduction des frontières du «Nous» national en répondant aux anxiétés identitaires des majoritaires ${ }^{43}$.

Sous l'effet de la restructuration néolibérale et de la peur de ne plus voir l'État prendre soin des siens, les majoritaires ${ }^{44}$ manifestent leur droit au statut de gestionnaires de l'espace national ${ }^{45}$. La construction des minorités comme objets de débats soumis à leur jugement est ici liée à volonté de restauration d'un sentiment de contrôle ${ }^{46}$. Cette approche permet de mettre en lumière l'ethnicité et la blanchité des majoritaires. Évidemment, la blanchité ne renvoie pas à l'existence d'une race blanche, mais bien à une construction sociale. Nous retenons la définition classique de Frankenberg: «First, whiteness is a position of structural advantage, of race privilege. Second, it is a "standpoint," a place from which people look at ourselves, at others, and at society. Third, whiteness refers to a set of cultural practices that are usually unmarked and unnamed ${ }^{47}$. La relation entre l'appartenance nationale et la blanchité n'est pas fixe et doit être étudiée empiriquement. Dans un contexte sociétal où la race et le terme "blancs» sont évacués du discours public, la blanchité peut être observée à travers les différentes pratiques qui construisent les minorités racialisées comme déviant de la norme nationale ${ }^{48}$. Comme le note Garner, de la question "d’où viens-tu» aux contrôles d'identité policiers, le fait que le terme «immigrant» colle ${ }^{49}$ aux corps des minorités racialisées nous force à reconnaître que l'appartenance 
Table 1. Nombre et proportion de commentaires positifs, négatifs et mitigés selon le média

\begin{tabular}{lccccccc}
\hline & TVA & JdeM & Radio-Can. & La Presse & Le Devoir & Total & $\%$ \\
\hline Positifs & $57(12,2 \%)$ & $37(16,2 \%)$ & 43 & 47 & 13 & $197(149)^{*}$ & 19,7 \\
& & & $(30,1 \%)$ & $(42,3 \%)$ & $(25 \%)$ & & $(16,1)^{*}$ \\
Négatifs & $390(83,3 \%)$ & 184 & 77 & 38 & 32 & 721 & 72,1 \\
& & $(80,7 \%)$ & $(54,6 \%)$ & $(34,2 \%)$ & $(61,5 \%)$ & $(701)^{*}$ & $(75,6)^{*}$ \\
Mitigés & 21 & 7 & 21 & 26 & 7 & 82 & 8,2 \\
& $(4,5 \%)$ & $(3,1 \%)$ & $(14,9 \%)$ & $(21,9 \%)$ & $(13,5 \%)$ & $(77)^{*}$ & $(8,3)^{*}$ \\
\hline Total & 468 & 228 & 141 & 111 & 52 & 1000 & 100 \\
& & & & & & $(927)^{*}$ & \\
\hline
\end{tabular}

* = commentaires de personnes catégorisées comme membres du groupe majoritaire

à la communauté nationale est inégalement distribuée par l'articulation avec l'ethnicité et la race ${ }^{50}$. D’ailleurs, dans le langage commun, la catégorie nationale «Québécois» est régulièrement ethnicisée et limitée au groupe majoritaire ${ }^{51}$.

Lanalyse vise ainsi à rendre compte du travail de patrouille des frontières nationales à l'endroit des réfugiés syriens en portant attention aux discours d'appartenance gouvernementale exprimés sur le mode du ressentiment et de l'entitlement ${ }^{52}$.

\section{Méthodologie et données}

Le corpus d'analyse compte 1000 commentaires publiés sur les pages Facebook publiques de cinq grands médias québécois, soit TVA Nouvelles, le Journal de Montréal, La Presse, Radio-Canada et Le Devoir ${ }^{53}$. Les quinze articles retenus ont été publiés entre septembre et décembre 2015, soit au moment où les débats politiques et la couverture médiatique sur les réfugiés syriens furent les plus intenses. Pour chaque média, trois articles ont été retenus, soit ceux qui ont généré le plus grand nombre de commentaires. Le nombre de commentaires est pondéré en fonction du volume pour chaque média. Conséquemment, léchantillon est à majorité composé de commentaires associés à des médias de type sensationnaliste ${ }^{54}$. Il a été construit en retenant les commentaires qui ont reçus le plus de «likes». Les résultats présentés ici se concentrent sur les individus catégorisés comme membres du groupe majoritaire ${ }^{55}$.

La méthode est inspirée de l'analyse critique de discours (CDA) et, plus spécifiquement, de l'approche discursive historique (DHA) de Ruth Wodak $^{56}$. Cela implique un positionnement épistémologique particulier: «CDA may be defined as fundamentally interested in analyzing opaque as well as transparent structural relationships of dominance, discrimination, power and control, as they are manifested in language $\aleph^{57}$. Cette approche a surtout été utilisée pour analyser le discours des élites, mais elle peut aussi être mise à profit pour étudier les discours des gens «ordinaires» sur une nouvelle plateforme discursive qui permet de mieux voir leur rôle actif au sein de l'ordre social inégalitaire ${ }^{58}$.

Les arguments des acteurs sont analysés en relation avec les «topoi» prépondérants, forme plurielle de «topos», concept renvoyant en rhétorique au lieu commun ${ }^{59}$. Ces thèmes et arguments communs sont au fondement de l'analyse de la présentation positive de soi et négative de l'Autre. Les données sont analysées en portant attention au déni du racisme à travers ce que Van Dijk (1998) appelle le carré idéologique par lequel la polarisation Nous/Eux est opérée, c'est-à-dire, d'un côté, l'accentuation des traits positifs et la diminution des points négatifs du Nous et, de l'autre, l'accentuation des traits négatifs et la diminution des points positifs des Autres ${ }^{60}$. Cela permet de voir comment l'exclusion racialisée est légitimée par des mécanismes discursifs qui font silence sur la race en misant notamment sur une présentation positive du Nous menacé et victimisé. L’analyse ne se limite donc pas aux commentaires ouvertement racistes ou hostiles. D'une part, l'accessibilité à ces derniers est limitée par le travail des modérateurs ${ }^{61}$. D’autre part, la méthodologie doit être adaptée au contexte marqué par l'idéologie hégémonique postulant le caractère post-racial de la société contemporaine ${ }^{62}$. Les outils de la DHA permettent donc d'analyser la racialisation dans les discours nationalistes en identifiant les arguments mobilisés dans le travail de patrouille des frontières.

\section{Analyse}

Les commentaires ont été classés comme étant positifs, négatifs ou mitigés et trois commentaires sur quatre sont négatifs. Cela ne renvoie pas nécessairement à une opposition catégorique à la réinstallation de réfugiés syriens au Canada, mais plutôt à un cadrage négatif de la situation. Concernant le profil positif, une part substantielle correspond à des commentaires dont le contenu témoigne d'une réaction à légard des commentaires jugés hostiles ou ignorants. Enfin, 8\% des données sont mitigées et tendent présenter l'accueil des réfugiés comme devant être conditionnelle à la préservation de la 
sécurité physique, culturelle ou économique des nationaux. Cinq principaux topoi sont identifiés: nombre, fardeau économique, responsabilité nationale, culture et danger. Étant donné les relations souvent étroites entre eux, ces séparations sont strictement analytiques. Il s'agit alors de bien saisir leurs logiques et dynamiques propres pour mieux comprendre les différentes stratégies argumentatives dans la représentation des réfugiés et des frontières nationales.

\section{Topos du nombre}

Les références aux nombres de réfugiés ainsi qu’aux qualificatifs associés ont pour effet de délégitimer le plan gouvernemental. Les individus parlent alors en termes de «milliers», de "beaucoup», d' "énorme», de "masse», etc. et ont largement recours au terme "trop». Dans certains discours mitigés, on lit par exemple que ce plan implique «trop» de réfugiés en "trop» peu de temps et qu'il dépasse donc la limite du raisonnable. Une pluralité dénoncés verse dans le catastrophisme et manifestent une crainte de menace pour le corps national. D’ailleurs, près d'un commentaire sur dix tend à dépeindre ces réfugiés comme faisant partie d'un afflux indésirable détrangers, ou pire, une invasion. Larrivée de 25000 personnes, représentant $0,7 \%$ de la population canadienne, apparaît alors comme étant la goutte qui fait déborder le vase. On comprend alors que le cadrage négatif $\mathrm{du}$ plan gouvernemental est indissociable de sentiments anti-immigration déjà présents.

Certains vont d'ailleurs jusquà affirmer que, couplée à l'immigration, l'arrivée de ces réfugiés aura pour conséquence une «expropriation» des majoritaires de «leur» pays. Par exemple:

[...] C'est rendu qu'on est plus chez nous dans nOtre propre pays... est ce qu'il y a des ministres qui s'en aperçoivent de ça!!?? Pour ma part je crois qu’on devrait commencer à savoir gérer les réfugiés qu'on a déjà au pays avant d’en laisser entrer d'autres (25000) [...] (Journal de Montréal 2, 16 likes).

Que l'expression d'une telle position se fasse dans un langage haineux, codé ou défensif, on voit bien que ceux qui s'inquiètent d'une «invasion» ayant pour effet de «déposséder» ou de réduire au statut de minorité la majorité ethnique blanche le font par la voie de l'autoreprésentation positive. D'une part, ils se positionnent comme étant ceux qui accueillent sur leur territoire des étrangers qui n'y appartiennent pas légitimement, reproduisant la frontière entre Eux et Nous. D'autre part, le Nous est présenté comme étant trop hospitalier envers Eux, les accueillant en trop grand nombre et menaçant ainsi lordre normal ${ }^{63}$. La dimension spatiale est ici centrale. Comme le souligne bien Hage, la qualité racialisante de ces discours est indissociable de l'appartenance nationale gouvernementale et donc d'une inquiétude quant au maintien du rôle de gestionnaire du chez soi national: "Clearly, what motivates the production of categories such as 'too many' in this context is the wish to construct or preserve not just a 'race', an 'ethnicity' or a 'culture', but also an imagined privileged relation between the imagined 'race', 'ethnicity' or 'culture' and the national space conceived as its own ${ }^{64}$.

\section{Topos du fardeau économique}

Un nombre important de commentaires représentent l'arrivée des réfugiés comme un fardeau pour les contribuables sous le mode du "qui va payer encore?» (TVA Nouvelles 1, 24 likes). Cet argument du fardeau est devenu un topos normal dans les débats occidentaux sur l'immigration. Il s'agit d'un des modes argumentatifs anti-immigration les plus sûrs, car il permet de situer la justification des postures restrictives à l'extérieur des considérations liées à la différence ethnique, raciale, religieuse, etc. Qui plus est, l'exclusion n'est pas présentée comme étant la résultante d'un manque de volonté, mais bien d'une impossibilité factuelle d'accueillir ${ }^{65}$.

La mobilisation d'arguments économiques participe à la patrouille des frontières en s'inscrivant dans la logique d'un nationalisme néolibéral différenciant les nationaux autonomes et productifs des Autres racialisés, dépendants, improductifs et profiteurs ${ }^{66}$. Les acteurs parlent de leur argent provenant de leurs taxes et impôts dépensés sans leur accord pour des «étrangers» non-méritants. Plusieurs anticipent des effets économiques négatifs et font référence au contexte économique saisi sous langle de la récession, de la croissance de la dette publique et des politiques d'austérité du gouvernement québécois. Nombreux sont ceux qui sétonnent donc que l'État puisse allouer des ressources à l'accueil de réfugiés dans une période de compressions budgétaires. Par exemple:

Ridicule!!! c'est trop 25 ooo personnes de plus d'un coup !!! voyons on arrête pas de ce serrer la ceinture à cause de l'austérité des gouvernements pis y parlent de couper les plus démunis pis y veulent faire v'nir du monde qui vont ce ramasser sur le Bs??? chercher l'erreur!!! (TVA Nouvelles 3, 55 likes).

Économiquement comment on fait pour se payer 25000 réfugier ... quand les gouvernement nous saigne au sang ... Y'as tu quelqu'un qui peux mettre un coût pour chaque réfugier ... (Journal de Montréal 2, 6 likes).

On peut ainsi faire valoir qu'il serait économiquement irresponsable de dépenser de l'argent que nous n'avons pas afin de venir en aide à des étrangers. En niant ou en mitigeant l'importance des préjugés et des stéréotypes, les acteurs revendiquent alors un ethos du raisonnable ${ }^{67}$. En mettant le focus 
sur le contexte économique, les individus sont à même de présenter leurs postures restrictives comme étant rationnellement fondées sur le monde réel et donc étrangères au racisme ${ }^{68}$.

Avons-nous les capacités? C'est la première question que nous devons poser ici. (La Presse 1, 3 likes).

[...] on a pu d'argent mais on accepté d'autre immigrant qui vont eux aussi avoir probablement besoin de tout les service ci haut mentionné je vais sûrement passé pour raciste mais non j’appelle ca vivre avec les moyen disponible rien a voir avec le pays d'où il viens!!! [...] (TVA Nouvelles 1, 242 likes).

Les réfugiés sont donc souvent déshumanisés en étant réduits à une dépense monétaire, ce qui est particulièrement frappant lorsque des personnes se questionnent à savoir quel sera le «coût» de chaque réfugié. En utilisant des formules du type "Je n'ai rien contre..., mais», "nous sommes tolérants..., mais» ou encore «nous voudrions aider, mais», les acteurs font référence à un contexte économique objectif qui permet de légitimer des pratiques d'exclusion sans faire appel à une rhétorique explicitement raciste. Il s'agit d'un cas classique de déni par présentation de soi positive. Le peuple québécois est représenté comme étant ouvert et généreux, mais nétant pas dans la mesure d'aider des étrangers dans le contexte actuel sans porter atteinte à son propre bien-être. À travers ce langage codé, on leur reproche ultimement d'abuser de l'hospitalité nationale. Ils sont d'ailleurs régulièrement accusés de chercher à profiter du système, notamment à travers la figure péjorative du «BS $»^{69}$.

Cette stigmatisation des réfugiés comme représentants de la mauvaise diversité acquiert une signification particulière dans le contexte local en fonction de l'image de l'immigrant idéal promue par l'État. Lévolution des politiques d'immigration suit effectivement la restructuration néolibérale en visant à maximiser l'immigration qualifiée et choisie par le privé, conçue comme une opportunité de compétition au sein de la globalisation tout en restreignant l'accès aux migrants jugés «indésirables ${ }^{70}$. Les discours faisant appel à des arguments de nature économique sont donc largement caractéristiques du nationalisme au sein de la conjoncture néolibérale où la désirabilité des étrangers est notamment évaluée en fonction de leur utilité et de leur autonomie individuelle ${ }^{71}$. Lanalyse montre que ces pratiques d'exclusion ne peuvent toutefois être réduites à une stricte rationalité économique en raison des articulations avec des sentiments de menace d'une autre nature, notamment culturelle et sécuritaire.

\section{Topos de la responsabilité}

Environ le quart des commentaires fait appel au topos de la responsabilité nationale. Nous faisons par-là référence à la stratégie argumentative qui met de l'avant la responsabilité des gouvernements à l'endroit de leur population. La logique veut que le gouvernement doive d'abord prendre soin de son peuple et donc resserrer les frontières ${ }^{72}$. Ce type de discours est particulièrement marqué par le ressentiment de majorités nationales blanches adoptant la perspective de victimes ${ }^{73}$. Cette auto-victimisation concerne la revendication des privilèges quant à l'accès aux ressources et l'affirmation du droit à un pouvoir symbolique privilégié en raison de l'appartenance à l' «entitled nation ${ }^{74}$.

Les autorités sont alors accusées de privilégier des étrangers et d'abandonner les «leurs». Les deux commentaires les plus populaires l'exemplifient:

accueillir des syriens et des réfugiés au canada ces complètement ridicule que le gouvernement commence donc par soccuper des Canadiens avant!!!!!!! (TvA Nouvelles 2, 1512 likes).

Il serait le temps que Le gourvernement Aide les sien avants D'aider les autres (TVA Nouvelles 1, 787 likes).

Il s'agit là d'une forme élémentaire de nationalisme banal où sont discriminés les Autres mis en opposition aux membres du «Nous» entendus comme membres de la communauté nationale imaginée, que ce "Nous» désigne l'ensemble de la communauté politique ou une certaine catégorie sociale ethniquement délimitée ${ }^{75}$.

La majorité des commentaires adopte une stratégie d'atténuation des traits négatifs du Nous ${ }^{76}$ suivant une formule selon laquelle aider les Autres peut constituer un principe digne, mais qui doit toujours rester secondaire par rapport à celui de la solidarité nationale, c'est-à-dire le devoir moral d'aider les siens. Même dans les appels à la fermeture des frontières, la justification première affichée est celle de la solidarité «entre nous». Cela rejoint les travaux d'Ahmed qui montrent que les discours racialisants de défense de la nation opèrent à travers une logique de légitimation qui n'affirment pas la haine de l'Autre, mais plutôt l'amour de $S i^{77}$. On retrouve d'ailleurs à plusieurs reprises l'expression «charité bien ordonnée commence par soi-même» et on souligne que l'on doit d'abord aider les «Québécois» ou encore régler "nos problèmes» avant de régler ceux des «autres». D'ailleurs, encore ici, des commentaires ont recours à des démentis qui permettent de nier la dimension raciste de l'exclusion. Par exemple, ce commentaire met l'accent sur la victimisation des Québécois:

Je ne suis pas raciste, je suis pour venir en aide aux gens MAIS LÀ en ce moment .... Ici c'est nous qui avons besoin d'aide [...] (TVA Nouvelles 1, 10 likes). 
Ces discours affirmant "Nous sommes le peuple» et «Nous d'abord» sont d'ailleurs centraux dans le répertoire des nationalismes populistes de droite occidentaux ${ }^{78}$. L'invocation du peuple peut s'avérer suffisante pour indiquer implicitement qui devrait occuper une position privilégiée au sein de la distribution des ressources et de l'attention étatique. Cela se voit dans l'opposition entre «nos Québécois d'ici, de chez nous" et les «immigrants», si bien que le fait de "venir d'ailleurs» apparaît incompatible avec l'identité québécoise et limite ainsi l'accès légitime aux ressources étatiques ${ }^{79}$. Limportance discursive de la territorialité est à saisir dans la logique de production de la nation où la différenciation entre les personnes qui se pensent natives et se voient comme familières et celles qui sont reconnues comme étrangères "out of place» est fonctionnelle au sentiment dêtre «chez soi» des premières ${ }^{80}$. La limite est donc souvent floue entre la revendication du droit à la priorité et l'exclusivité en matière de distribution des ressources. Lélément central est le fait que les majoritaires définissent les conditions de l'entitlement en fonction de l'appartenance nationale ${ }^{81}$. En fait, ces individus demandent que l'État «commence» par s'occuper des nationaux, mais il n'est pas clair à savoir s'il serait ultimement acceptable d'aider ces étrangers. La compétition pour les ressources de l'État est aussi une compétition pour ses soins et son attention et est paradigmatique de la culture de l'inquiétude caractéristique de la relation individu-société nationale sous la restructuration néolibérale et commandant une sécurisation des frontières ${ }^{82}$.

La représentation racialisée des réfugiés comme nonméritants est appuyée par le recours à 4 figures de «vrais nationaux » abandonnés ou désavantagés par l'État, soit les travailleurs et familles pauvres, les itinérants, les enfants et les aînés. Par exemple:

mais de toute façon le gouvernement Couillard se fou royalement de l'a'vis des québécois. Il préfère prendre notre argent des impôts et des taxes pour des réfugiés plutot que de mettre notre argent dans léducation pour les enfants québécois ou bien dans le service de santé. Il y a des enfants québécois qui ne mangent pas à leur faim tous les jours et qui ont froid l'hiver pour manque de manteau et de bottes. Il y a des sans abris québécois qui meurent de faim et de froid tout l'hiver!!! qu'est ce que le gouvernement fait pour tous ces gens québécois ????? (La Presse 2, 5 likes).

Ces figures sont généralement précédées du déterminant possessif «nos», si bien que la nation est représentée comme un chez soi familial auquel les réfugiés syriens ne sauraient appartenir $^{83}$. On retrouve donc, d'un côté, nos gens dans le besoin, de chez nous, de notre peuple et, de l'autre, la pauvreté des autres, racialisées comme n'appartenant pas «naturellement» au territoire national. Les individus tendent effectivement à se représenter comme appartenant à l'entitled nation où le mérite quant à l'accès aux ressources est déterminé en fonction de la réponse à la question «que fais-tu?", mais aussi et surtout à la question «qui es-tu? $»^{84}$. Cela va dans le sens de nombreuses études qui montrent que l'appui aux politiques de redistribution de l'État social est influencé par les perceptions racialisées à savoir qui bénéficie des ressources étatiques ${ }^{85}$. D'ailleurs, nos analyses rejoignent aussi le constat que la solidarité est limitée par la perception du mérite en fonction de la nationalité, de la religion, de l'ethnicité et de la race et que le welfare chauvinism est structuré par les perceptions de menace culturelle à la nation ${ }^{86}$.

\section{Topos de la culture}

Les commentaires associés à ce topos postulent souvent l'impossibilité ou le refus de l’intégration des réfugiés à la culture nationale, intégration d'ailleurs largement entendue comme assimilation à la norme majoritaire. Si les réfugiés ne sont pas nécessairement toujours catégorisés comme musulmans, il reste qu'ils sont souvent représentés à travers un prisme influencé par le climat d'islamophobie ambiant. Ils ont d'ailleurs tendance à être amalgamés avec les musulmans présents sur le territoire national et à être perçus comme aggravant le travail de "gestion» de la diversité. Par exemple: «Bravo, on a déjà de la misère à gérer ceux que l’on a déjà!» (Journal de Montréal 1, 4 likes). D’une part, la faute est mise sur les différences culturelles "objectives» sélectionnées pour le marquage des frontières. En ce sens, les réfugiés seraient «en soi» si différents de la culture occidentale que leur intégration est présentée comme objectivement impossible. D’autre part, au niveau subjectif, si on reconnaît une agentivité aux réfugiés et aux musulmans plus largement, on met en cause leur volonté. Ce faisant, l'exclusion n'est pas présentée comme étant légitime en raison d'une quelconque hostilité provenant de la part des «nationaux». Ce sont au contraire les réfugiés eux-mêmes qui sont rendus responsables en raison de leur refus postulé de faire des efforts d'intégration:

nous n’en voulons pas ils ne veulent pas s'intégré a notre pays (Journal de Montréal 1, 4 likes).

Calvince on en a assez de même ont a pas les moyens et en plus ils veulent pas s'adapter?? (TVA Nouvelles 1, 139 likes).

Un autre type de formulation consiste à présenter les réfugiés comme ingrats et ne respectant pas la culture de la terre d’accueil, s'appuyant sur le stéréotype racialisant du musulman intransigeant hostile à notre mode de vie libéral occiden$\mathrm{tal}^{87}$. Il s'agit là d'une forme de ressentiment blanc devenue commune au sein de plusieurs sociétés occidentales où les immigrants, réduits au statut d'«invités», sont opposés aux 
"hôtes» nationaux qui défendent ce qui leur appartient ${ }^{88}$. Les Autres ne sont alors pas seulement accusés de vouloir maintenir leurs habitudes culturelles ou de vivre dans une société parallèle. Ils incorporent plutôt une menace culturelle. C'est là que s'exprime le plus clairement un nationalisme de type défensif au sens entendu par Hall (1993) où l'appartenance culturelle fonctionne comme un langage codé pour la race. On fait alors appel à la nécessité de défendre «nos valeurs", «nos mœurs» et «nos coutumes». Ici, la dimension raciste de l'exclusion est donc discursivement niée par la représentation de l'Autre comme abusant de notre hospitalité et de notre tolérance. Le déni opère alors à travers le mécanisme de présentation positive de soi et négative de l'Autre: «Nous» sommes trop tolérants envers ceux qui sont les «vrais» intolérants, les musulmans qui menacent la culture nationale.

La stratégie argumentative justifiant l'exclusion par l'adoption d'une posture défensive s'accompagne aussi de la rhétorique de l'islamisation de l'Occident liée à un régime de représentation racialisée inscrit dans des relations de pouvoirs globales. Les commentaires analysés puisent dans un savoir orientaliste où, par répétition d'images et de discours, des significations stigmatisantes "collent» aux corps d'individus cherchant refuge ${ }^{89}$. Le musulman est alors présenté comme irrationnel et fanatique. Il s'agit ici d'exemples de la stratégie du racisme inversé où les réfugiés sont dépeints comme des islamistes radicaux anti-occidentaux:

Ce que l'on sait par contre, c'est que dans 20 ans. Ils seront plus nombreux que nous, éliront leurs députés. Prendront le contrôle et feront disparaître notre culture. Ça, on le sait! (TVA Nouvelles 3, 9 likes).

le canada peut meme pas faire vivre sont peuple quoi il vont faire avec 25000 syriens qui veut toute changer le mondes avec leur religion (Radio-Canada 1, 2 likes).

1ere vague du Tsunami islamiste qui rentre au Canada ... tout un cheval de Troie, qui vient de rentrer dans nos portes ...!!! (TVA Nouvelles 2, 232 likes).

Certains avancent aussi que les pays occidentaux ne devraient pas être responsables de ces réfugiés en arguant que ce sont les États arabes et musulmans qui devraient les prendre en charge. On retrouve ici la production de ce que Saïd appelait la division géographique imaginaire qui soutient la binarité Occident/Orient ${ }^{90}$. La représentation d'un bloc islamique homogène est ainsi fonctionnelle à la consolidation d'une identité civilisationnelle occidentale.

La racialisation des réfugiés syriens comme menace culturelle est étroitement liée à la dénonciation des élites politiques, dites soumises devant les musulmans, que ce soit au nom du multiculturalisme canadien, à des fins électoralistes ou dans une visée fédéraliste anti-québécoise. Par exemple:

Le Parti Libéral est l'outil d'assimilation au multiculturalisme du Parti Libéral du Canada. Politique de racolage ethnique à des fins purement électorales. (Le Devoir 3, 4 likes).

Nos données supportent l'analyse de Bilge (2013) qui constate une légitimation de la figure du «white worrier»; le "Nous» national racialement codé est posé en position de victime face à la menace de la «différence» culturelle de l'Autre promue par lélite multiculturaliste déconnectée des inquiétudes de la majorité silencieuse. Cela rejoint aussi de nombreuses études qui montrent que les majoritaires peuvent adopter des stratégies discursives de patrouille des frontières entre "vrais» nationaux et étrangers en évitant la catégorisation raciste directe pour plutôt cibler les élites accusées de verser dans le "politiquement correct», de favoriser de façon indue les étrangers et de censurer les inquiétudes de la majorité (blanche ${ }^{91}$.

\section{Topos du danger}

Environ le quart des commentaires cadrent l'arrivée des réfugiés syriens comme un risque pour la sécurité nationale. Ces discours qui amalgament explicitement ou implicitement les termes "réfugiés», "musulmans » et "terroristes » s'inscrivent dans un registre caractéristique de l'«islamophobie globale» post-200192. La représentation racialisée et genrée de l'homme musulman violent et dangereux est à ce propos centrale ${ }^{93}$. Les discours sur les réfugiés syriens déployés sur les médias sociaux donnent lieu à des représentations stigmatisantes de la masculinité des hommes moyen-orientaux. En effet, ils sont tantôt dépeints comme étant des terroristes, donc de violents guerriers, et tantôt comme des lâches abandonnant leur patrie et leurs femmes ${ }^{94}$. Cela sert aussi bien des postures de fermeture des frontières que des revendications d'inclusion sélective qui appellent à l’admission exclusive des femmes et enfants ou à un renforcement des protocoles de sécurité ${ }^{95}$. Compte tenu du risque compris comme étant inhérent, ces personnes utilisent la catégorie du «faux-réfugié» et cherchent à légitimer l'exclusion en la présentant comme appartenant au domaine du raisonnable. La rhétorique du "faux-réfugiés", qui sest développé au sein du discours politique conservateur et dans les médias, est à inscrire dans une dynamique de sécuritisation de l'immigration au Canada depuis les années $1990^{96}$. L'idée selon laquelle des migrants abuseraient de la générosité et de l'hospitalité du Canada a notamment été promue par le gouvernement Harper en 2012 dans le cadre de réformes qui ont limités les droits des demandeurs d'asile ${ }^{97}$. Sans nécessairement procéder à une démonisation 
de l'ensemble des Syriens, une hiérarchie de la valeur des vies est établie. En effet, l'invocation du risque sécuritaire dans lopposition à la réinstallation de personnes en situation d'insécurité en raison d'une guerre civile signifie que ce n'est pas la sécurité qui est la priorité, mais bien «notre» sécurité:

On a de l'intolérance car on a peur que sur les 25000 a 125000 réfugiés qu'il en aille une 50 aine qui pourrait faire ben du dommage. Protegeons nous avant de protéger les autres (Journal de Montréal 2, 6 likes).

Les réfugiés syriens sont aussi accusés par certains dêtre partie prenante d'un plan d'invasion djihadiste. Ils sont aussi racialisés en étant dépeints comme imprégnés d'une culture guerrière, étrangère et hostile à la culture occidentale:

Les musulmans sont en guerre depuis la nuit des temps, en guerre entre eux, contre les femmes, contre tous ceux qui ne sont pas de la même religion qu'eux. [...] En vivant ici, ils reproduisent ce qu'ils ont fui chez eux, la guerre et les conflits tout le temps. Est ce que c'est ce que nous voulons? [...] (Le Devoir 1, 1 like).

Ça va tellement nous péter dans face... Vous arrêtez pas de brailler que les immigrants ne s'adaptent pas à nos règles de vie et soudain, vous voulez accueillir à bras grands ouverts 10 ooo immigrants qui $\mathrm{n}$ `ont connu que la guerre et la violence. Par quel moyen vous croyez qu'ils vont revendiquer quoi que ce soit après ça? De la seule manière qu'ils ont appris: la violence. Ils ont beau être humains comme nous, ils n’ont pas en eux les concepts de démocratie, liberté et égalité; ils n'ont pas déducation et ils n'ont surtout pas les mêmes priorités que les Européens ou américains. (TVA Nouvelles 1, 52 likes).

La présentation négative des musulmans comme violents et belliqueux ${ }^{98}$ trouve son pendant dans la présentation positive de soi comme peuple pacifique. La dimension raciste est niée par la rhétorique de l'auto-défense: ce n'est pas que nous les détestons, c'est que nous voulons protéger les «nôtres» et «notre» quiétude. Les commentateurs s'en prennent donc aux autorités politiques à qui on demande d'agir en respect de ce que Walters appelle la domopolitique, c'est-à-dire une représentation du territoire national comme "home» familial devant être sécurisé face à ceux qui n'y appartiennent pas naturellement. ${ }^{99}$

Nos données montrent donc une forte importance du cadrage des réfugiés sous l'angle du danger, les commentaires allant de l'expression d'inquiétudes sans référence à l'Islam à des énoncés faisant preuve d'une islamophobie ouverte et dépeignant les réfugiés comme des terroristes. D’ailleurs, la construction des réfugiés comme menace sécuritaire ne peut être saisie qu'en rapport avec la race et les hiérarchies issues de la modernité coloniale. ${ }^{100}$ Elle est effectivement partie prenante d'un imaginaire racialisé où toutes les populations étrangères ne sont pas interprétées comme impliquant le même degré de dangerosité. Les individus perçus comme appartenant à l'Islam sont effectivement situés comme étant ceux qui sont les plus «à risque ${ }^{101}$. Les discours de sécurisation sont structurés par le contexte de guerre contre le terrorisme. Les images du terroriste et du musulman sont collées (stuck together) et quiconque «ressemble» à l'idée de l'ennemi est soumis au regard suspicieux ${ }^{102}$. Les discours analysés ici sont aussi performatifs. Ils puisent dans le répertoire de stéréotypes partagés et réactualisent la figure de l'altérité menaçante. Les commentaires sur la possibilité d'infiltration de terroristes parmi les réfugiés rejoignent les travaux de Ahmed. La narration du «terroriste potentiel» a pour effet de restreindre la mobilité des corps qui sont lus comme étant associés à l'idée contemporaine du terrorisme.

\section{Conclusion}

Les pratiques discursives analysés ici permettent de voir que les dynamiques d'inclusion/exclusion se déploient au sein d'une relation triangulaire. Au niveau des stratégies référentielles, trois catégories d'acteurs sont donc centrales. Il s'agit du «peuple», des «élites politiques» et des «étrangers». Pour la première, au-delà de l'utilisation récurrente du pronom «Nous», on retrouve les termes «Québécois» et, dans une moindre mesure, "Canadiens» auxquels sont parfois ajoutés des qualificatifs signalant une compréhension exclusive de ces identités, par exemple «dorigine», «de souche» et «vrais». Dans la construction des frontières du Nous par les majoritaires ordinaires, les significations se rattachant à la nation, à l'ethnicité et à la blanchité doivent être déduites du langage de la pratique ${ }^{103}$. En effet, les acteurs nont pas explicitement recours à ces catégories dans leurs pratiques discursives quotidiennes. Le groupe «canadien-français» nest pas nommé en tant que tel, mais notre interprétation suggère que les références à "Nous les Québécois» tendent à signifier la majorité ethnique blanche, ce qui peut notamment être déduit des discours de préservation comme dans l'opposition entre les termes "Québécois» et «immigrants». Rappelons que, suivant notre méthodologie, la blanchité et l'ethnicité des commentateurs reposent sur une déduction. La représentation du «Nous» comme accueillant et tolérant, mais inquiet en raison de sa fragilité culturelle et des menaces externes permet de légitimer les exclusions racialisées tout en niant sa dimension raciste. Rappelons toutefois que, suivant notre méthodologie, la catégorisation des commentateurs comme membres du groupe majoritaire est faite en fonction de leur nom et ne répond pas d'une autoidentification par ceux-ci.

Le second groupe identifié est celui des réfugiés syriens. Nombreux sont ceux qui adoptent les termes utilisés dans les discours médiatiques et politiques, en loccurrence «les 
réfugiés» ou «les Syriens». Par contre, d'autres termes sont utilisés pour marquer leur altérité, notamment ceux d' "étrangers», de gens "d'ailleurs», de musulmans et d'immigrants. Lamalgame récurrent avec les immigrants signifie que les réfugiés syriens sont régulièrement vus comme étant destinés à demeurer à l'extérieur des frontières de la communauté nationale puisque la résidence sur le territoire ne garantirait pas l'appartenance et la reconnaissance comme membres de la communauté nationale. Au niveau des caractéristiques attribuées, si plusieurs reconnaissent que les membres de ce groupe cherchent refuge en raison de la guerre sévissant dans leur pays, les réfugiés syriens se voient qualifiés d'ingrats, de dangereux, de potentiels terroristes, d'islamistes radicaux, de barbares, de prémodernes, de lâches et de profiteurs des ressources et services étatiques. Ils sont aussi marqués par leur différence culturelle/civilisationnelle, c'est-à-dire qu'ils sont représentés comme provenant d'une région aux mœurs et valeurs très éloignés, sinon incompatibles avec la civilisation occidentale moderne. Les réfugiés syriens font alors souvent office de menace économique, culturelle et sécuritaire pour la nation québécoise. Nous avons pu voir qu’aussi bien des stratégies d'intensification que de mitigation étaient employées. Si des discours hostiles et ouvertement racistes ou xénophobes sont bien présents, ils le sont toutefois moins que ce que l'on pourrait attendre à première vue de la part d'usagers des médias sociaux.

Les autorités politiques multiculturalistes sont quant à elles présentées comme étant caractérisées par leur richesse, leur déconnexion du «vrai peuple» et leur soumission aux «immigrants» et aux musulmans. Les libéraux au pouvoir sont les principaux destinataires du ressentiment blanc. En regard des stratégies d'intensification ou de mitigation, les discours s'adressant aux élites politiques se démarquent souvent par leur forte hostilité, les individus nétant pas gênés d'exprimer leur rejet de ceux qui sont vus comme des traitres. Cette hostilité exprimée peut d'ailleurs être plus grande à l'endroit des élites que des réfugiés. Cela rejoint une stratégie argumentative courante dans les discours nationalistes populistes de droite qui exploitent et exacerbent le ressentiment des classes moyennes et populaires où les références aux outsiders peuvent demeurer implicites en s'en prenant à la traîtrise des élites déracinées ${ }^{104}$. Il ne semble pas fortuit que l'immigration ait été un thème central des élections québécoises d’automne 2018 remportées par la Coalition Avenir Québec (CAQ). Le nouveau premier ministre François Legault a notamment soutenu que la capacité d'intégration du Québec était dépassée et que les Québécois étaient «tannés» de se faire donner des "leçons» par les libéraux qui les accuseraient dêtre intolérants envers les immigrants et les minorités. Dans un contexte de pénurie de main d'œuvre, il a notamment annoncé une réduction l'immigration de plus de $20 \%$, incluant la catégorie des réfugiés, et l'expulsion des immigrants qui échoueraient à des tests de français et de valeurs trois ans après leur établissement ${ }^{105}$. Le ressentiment nationaliste exprimé sur les médias sociaux trouve donc une résonance avec le discours caquiste d'affirmation nationale qui martèle que les "Québécois» ont le droit de vouloir mieux contrôler leurs frontières.

\section{Notes}

1 Si la publication de cette photo a eu des échos partout à l'international, la mort du petit Alan est liée au Canada dans la mesure où la demande de parrainage de sa tante vivant au Canada a été rejetée par les autorités canadiennes. Ce refus aurait poussé les membres de la famille Kurdi, alors en Turquie, à entreprendre la traversée dangereuse en bateau. On apprendra plus tard que la demande refusée ne concernait pas le père d'Alan, mais bien son oncle. Il reste que ce refus aurait encouragé l'adoption d'une voie de migration dangereuse.

2 Le parti conservateur, alors au pouvoir, maintient alors une approche restrictive justifiée par un argumentaire sécuritaire et économique en phase avec les modifications apportées sous sa gouverne au régime de citoyenneté et à la politique d'immigration. Voir Mathieu Forcier et Frédérick-Guillaume Dufour, "Immigration, Neoconservatism and Neoliberalism: The New Canadian Citizenship Regime in the Light of European Trajectories", Cogent Social Sciences 2, no 1 (2016): 1-18; Yasmeen Abu-Laban, «Building a New Citizenship Regime? Immigration and Multiculturalism in Canada», in Citizenship in Transnational Perspective, dir. Jatinder. Mann (New York: Palgrave Macmillan, 2017), 263-82.

3 "Canadians Divided along Political Lines over Whether to Accept Thousands of Refugees in Current Crisis», Angus Reid Institute, 2015, http://angusreid.org/wp-content/ uploads/2015/09/2015-09-04-Migrants.pdf.

4 Louise Leduc, «Six Québécois sur dix disent non aux réfugiés», La Presse, 2015.

5 Nous préférons la définition proposée par Alana Lentin à celle classique de Robert Miles puisqu'elle ne se limite pas à la biologisation: "Racialization involves endowing the characteristics, appearances, traditions, and lifestyles attributed to groups of different "others" with negative signifiers that are deemed to be natural and insurmountable. [...] It puts into words the very thing about a particular group that is said to disturb us and pose a threat to our way of life». Alana Lentin, Racism and Ethnic Discrimination (New York: Rosen Publishing Group, 2011).

6 Au cours des dernières années, le Québec accueillait environ 50 ooo nouveaux résidents. Le total au niveau canadien est d'environ 300 ooo et le gouvernement actuel s'est donné une cible de 350 ooo pour 2021. En 2016, la part des immigrants au Québec dans l'ensemble du Canada était de $18 \%$ 
et donc inférieure au poids démographique de la province qui est lui de $23 \%$. Le Québec a la responsabilité exclusive de la sélection (excluant le regroupement familial et les réfugiés), de l'accueil et de l'intégration linguistique, culturelle et économique. Les immigrants économiques sont sélectionnés en fonction de critères tels que lâge, la formation, l'expérience professionnelle, l'autonomie financière, les connaissances linguistiques, etc. En vertu de l'Accord Canada-Québec (1991), le Québec est tenu d’accueillir un nombre de personnes réfugiés correspondant à son poids démographique au sein du Canada.

7 Alors que la proportion de ceux favorables à une réduction de l'immigration était en baisse constante entre 1993 et 2006 - de 52,1\% à 19,7\%, elle est en hausse soutenue depuis, sétablissant à $28,8 \%$ en 2011, une augmentation sétant produite dans un contexte marqué par la crise financière et la «crise» des accommodements raisonnables (Turgeon et Bilodeau, 2014: 324). En 2018, dans un contexte économique favorable marqué par une pénurie de main-d’œuvre, environ $45 \%$ des Québécois et un francophone sur deux étaient d'avis qu'il y a trop d'immigrants. Voir Luc Turgeon et Antoine Bilodeau, "Minority Nations and Attitudes towards Immigration: The Case of Quebec», Nations and Nationalism 2O, no 2 (2014); «La politique provinciale au Québec», Léger, 2018, http://storage.quebecormedia. $\mathrm{com} / \mathrm{v1} /$ tvanouvelles_prod/file/oa8o8e18-269o-40c3-b6fb4 febab2ao620_PolitiqueprovincialeQC-Avril2018FINALTVA.PDF; «Sondage Ipsos-La Presse. L'immigration au Québec», Ipsos, 2018, https://www.ipsos.com/fr-ca/news-polls/ la-presse-immigration-au-quebec.

8 La société québécoise a été marquée, au moins depuis 2006, par de nombreux débats sociaux sur l'immigration, l'identité nationale, l'intégration et la diversité religieuse. Tous ont participé à la problématisation de la présence musulmane sur le territoire québécois. De nombreux travaux ont montré que ceux-ci ont eu pour effet de banaliser les discours xénophobes et racistes (en particulier islamophobes) de la part des Québécois d’origine canadienne-française et que les postures d'exclusion étaient énoncées en référence à la fragilité de leur culture nationale. Voir Darryl Leroux, "Québec Nationalism and the Production of Difference: The Bouchard-Taylor Commission, the Hérouxville Code of Conduct, and Québec's Immigrant Integration Policy», Québec Studies 49 (2010); Maryse Potvin, "Relations ethniques et crise des "accommodements raisonnables" au Québec", dans Managing Immigration and Diversity in Canada. A Transatlantic Dialogue in the New Age of Migration, dir. Dan Rodríguez-García (Montréal et Kingston: McGill-Queen’s University Press, 2012).; Sirma Bilge, «Reading the Racial Subtext of the Québécois Accommodation Controversy: An Analytics of Racialized Governmentality», Politikon 40, no 1 (2013); Frédérick Nadeau et Denise Helly, «Extreme Right in Quebec? The Facebook Pages in Favor of the "Quebec Charter of Values" ", Canadian Ethnic Studies 48, no 1 (2016); Leïla Benhadjoudja, «Laïcité narrative et sécularonationalisme au Québec à l'épreuve de la race, du genre et de la sexualité», Sciences Religieuses 46, no 2 (2017).

9 Une étude de Wright et al. montre que les Québécois francophones ont, dans l'ensemble, des attitudes plus exclusives à l'endroit des musulmans que les Canadiens anglophones et les États-Uniens. Cela se manifeste dans les opinions par rapport à l'opposition au port du hijab et du niqab, à lacceptation de la moquerie du prophète Mohammed dans les journaux et du rejet du temps de piscine réservé pour les femmes musulmanes. Pour les auteurs, le fait que la tolérance à l'endroit des musulmans soit moins importante au Québec s'explique partiellement par le sécularisme de la province. Par exemple, le port de la croix est aussi moins bien vu que dans les contextes de comparaison. Toutefois, les traitements différenciés en fonction des appartenances religieuses, toujours plus défavorables aux musulmans, indiquent que la stéréotypification de ces derniers est un facteur dexplication tout aussi important. Voir Matthew Wright, Richard Johnston, Jack Citrin et Stuart Soroka, «Multiculturalism and Muslim Accommodation: Policy and Predisposition across Three Political Contexts", Comparative Political Studies 50, no 1 (2017): 125. 44\% des Québécois seraient d'avis que l'immigration musulmane devrait être interdite. Voir Katia Gagnon, «Les intolérants », La Presse, 2016, http://plus.lapresse.ca/screens/db3696dd4a1b-4972-a6a3-d6774797a6fc\%7CeisdhnJ1TBxJ.html. Une majorité affirme avoir une opinion négative des musulmans. Voir Louise Leduc, "Le malaise musulman», La Presse, 2014, http://plus.lapresse.ca/screens/4do2-dc6853237404-ad87-7128ac1c6068_7C__o.html. Une majorité de Québécois estiment que les musulmans sont mal intégrés, s’opposent à la présence de mosquées, considèrent le voile islamique comme un signe de soumission et sont inquiets quant à la présence de musulmans en lien avec les questions de sécurité intérieure. Sur l’ensemble de ces questions relatives à la présence musulmane, les Québécois sont généralement relativement plus exclusifs que les autres Canadiens. Voir «Les Canadiens, le populisme et la xénophobie», CROP, 2017, https://ici.radio-canada.ca/nouvelles/ special/2017/03/sondage-crop/Sondage\%20crop-RadioCanada.pdf.

10 Il devient alors de plus en plus difficile de départager franchement la droite conservatrice de celle radicale puisque les rhétoriques populistes de défense face aux menaces migratoires et musulmanes deviennent les lieux communs des politiques nationalistes. Voir Ferruh Yilmaz, «RightWing Hegemony and Immigration: How the Populist Far-Right Achieved Hegemony through the Immigration Debate in Europe », Current Sociology 60, no 3 (2012); Aristotle Kallis, «Breaking the Taboos and "Mainstreaming" the Extreme: The Debates on Restricting Islamic Symbols in Europe", dans Right-Wing Populism in Europe: Politics and Discourse, dir. Ruth Wodak, Majid Khosravinik et Brigitte Mral (Londres: Bloomsbury Academic); Ulrike 
M. Vieten et Scott Poynting, «Contemporary Far-Right Racist Populism in Europe», Journal of Intercultural Studies 37, no 6 (2017); Cas Mudde, «Europe's Centre Right Is on the Wrong Track with "Good Populism" ", Guardian, 2018, https://www.theguardian.com/commentisfree/2017/ oct/3o/europe-centre-right-wrong-track-good-populismnativism.

11 Gurminder K. Bhambra, «Brexit, Trump and "Methodological Whiteness" ", British Journal of Sociology 68, no S1 (2017); Sivamohan Valluvan, «Defining and Challenging the New Nationalism ", Juncture 23, no 4 (2017); Satnam Virdee et Brendan McGeever, "Racism, Crisis, Brexit», Ethnic and Racial Studies 41, no 10 (2018).

12 Par "majoritaire ordinaire», nous faisons référence aux Québécois d'ethnicité canadienne-française de façon générale. Le terme «ordinaire» ne se veut donc pas péjoratif. D’ailleurs, l'auteur appartient lui-même à cette catégorie identitaire. Le choix de ce terme est justifié par le fait que la présente étude se distingue de celles qui analyse les discours des élites politiques et médiatiques.

13 La race et la blanchité sont des constructions sociales structurantes. Suivant Hage, la blanchité n'est donc pas une essence, mais bien une position fantasmée de dominance culturelle héritée de la colonisation européenne. Comme le note Thobani, au Canada comme dans les autres sociétés de peuplement blanc, la blanchité correspond à l'identité nationale positivement racialisée et confère un sentiment de «managerial entitlement» sur les non-blancs. Sunera Thobani, Exalted Subjects. Studies in the Making of Race and Nation in Canada (Toronto: University of Toronto Press, 2007).

14 C'est donc dire qu'il est question d'un espace où les majoritaires peuvent reproduire les frontières identitaires et le statu quo en ce qui a trait à la distribution inégale du pouvoir et des privilèges.

15 Deborah Lupton, Digital Sociology (Londres: Routledge, 2015); Andrew Jakubowicz, "Cyber Racism», dans More or Less: Democracy and New Media, dir. Helen Sykes (Melbourne: Future Leaders, 2012).

16 Irfan Chaudry, «\#Hashtagging Hate: Using Twitter to Track Racism Online», First Monday 20, no 2 (2015); Nadeau et Helly, «Extreme Right in Quebec? The Facebook Pages in Favor of the "Quebec Charter of Values" "; Osmud Rahman, Benjamin Fung et Alexia Yeo, «Exploring the Meanings of Hijab through Online Comments in Canada", Journal of Intercultural Communication Research 45, no 3 (2016): 214-32; Maryse Potvin, "Discours racistes et propagande haineuse. Trois groupes populistes identitaires au Québec», Diversité urbaine 17 (2017): 49-72.

17 Voir Les Back, «Aryans Reading Adorno: Cyber-Culture and Twenty-First Century Racism ", Ethnic and Racial Studies 25, no 4 (2002): 628-51; Phyllis B. Gerstenfeld, Diana R. Grant et Chau-Pu Chiang, "Hate Online: A Content Analysis of Extremist Internet Sites", Analyses of Social Issues and Public Policy 3, no 1 (2003): 29-44; Jessie Daniels,
"Race, Civil Rights and Hate Speech in the Digital Era», dans Learning Race and Ethnicity. Youth and Digital Media, dir. Anne Everett, 129-54 (Cambridge: MIT Press, 2008); Jessie Daniels, Cyber Racism: White Supremacy Online and the New Attack on Civil Rights (Lanham: Rowman \& Littlefield Publishers, 2009); Yaman Akdeniz, Racism on the Internet (Strasbourg: Council of Europe Publishing, 2009); Chris Allen, "Opposing Islamification or Promoting Islamophobia? Understanding the English Defence League", Patterns of Prejudice 45, no 4 (2011): 279-94; Manuela Caiani et Patricia Kröll, «The Transnationalization of the Extreme Right and the Use of the Internet», International Journal of Comparative and Applied Criminal Justice 39, no 4 (2015): 331-51; Inari Sakki et Katarina Pettersson, «Discursive Constructions of Otherness in Populist Radical Right Political Blogs», European Journal of Social Psychology 46, no 2 (2016): 156-70; Jamie Cleland, Chris Anderson et Jack Aldridge-Deacon, «Islamophobia, War and Non-Muslims as Victims: An Analysis of Online Discourse on an English Defence League Message Board », Ethnic and Racial Studies 41, no 9 (2017): 1541-57.

18 Nous retenons la définition du racisme formulée par Balibar qui, en le conceptualisant comme étant pluriel, permet de voir que sa réduction au racisme biologique sert d’alibi aux nationalistes visant l'immigration: «Le racisme - véritable "phénomène total" - s'inscrit dans des pratiques (des formes de violence, de mépris, d'intolérance, d'humiliation, d'exploitation), dans des discours et des représentations qui sont autant délaborations intellectuelles du fantasme de prophylaxie ou de ségrégation (nécessité de purifier le corps social, de préserver l'identité du "soi", du "nous", de toute promiscuité, de tout métissage, de tout envahissement), et qui sarticulent autour des stigmates de l'altérité (nom, couleur de peau, pratiques religieuses)», Étienne Balibar, "Y a-t-il un "néo-racisme"?", dans Race, nation, classe. Les identités ambiguës, dir. Étienne Balibar et Immanuel Wallerstein (Paris: La Découverte, 1997).

19 Qui plus est, l'expression de discours plus «modérés» est susceptible de générer davantage d'appuis de la part des autres participants en ligne et de normaliser les pratiques racialisantes. Voir Adam Klein, "Slipping Racism into the Mainstream: A Theory of Information Laundering", Communication Theory 22, no 4 (2012): 427-48; Nicholas Faulkner et Ana-Maria Bliuc, " "It's Okay to Be Racist": Moral Disengagement in Online Discussions of Racist Incidents in Australia ", Ethnic and Racial Studies 39, no 14 (2016): 2545-63.

20 Matthew W. Hughey et Jessie Daniels, «Racist Comments at Online News Sites: A Methodological Dilemma for Discourse Analysis », Media, Culture \& Society 35, no 3 (2013): $332-47$.

21 Heather Hensman Kettrey et Whitney Nicole Laster, "Staking in the "World White Web": An Exploration of the Roles of Overt and Color-Blind Racism in Maintaining Racial Boundaries on a Popular Web Site», Social Currents 
1, no 3 (2014): 257-74; Matthew W. Hughey, «Show Me Your Papers! Obama's Birth and the Whiteness of Belonging", Qualitative Journal 35, no 35 (2012): 2; Hughey et Daniels, "Racist Comments at Online Bews Sites »; Theresa Senft et Safiya Umoja Noble, "Race and Socia Media,» dans The Social Media Handbook, dir. Jeremy Hunsinger et Theresa Senft (New York: Routledge, 2014).

22 Simon Goodman, "Constructing Asylum Seeking Families», Critical Approaches to Discourse Analysis across Disciplines 1, no 1 (2007): 36-50; Fiona H. McKay, Samantha L. Thomas et Warwick Blood, " Any One of These Boat People Could Be a Terrorist for All We Know!" Media Representations and Public Perceptions of "Boat People" Arrivals in Australia», Journalism 12, no 5 (2011): 607-26; Shani Burke et Simon Goodman, "Bring Back Hitler's Gas Chambers": Asylum Seeking, Nazis and Facebook - A Discursive Analysis ", Discourse \& Society 23, no 1 (2012): 19-33; Isabel Alonso Belmonte, Daniel Chornet et Anne McCabe, «Ideological Stances in Internet Users' Discursive Construction of Immigration, Race and Racism: An Online Newspaper Case Study", dans Discourses on Immigration in Times of Economic Crisis: A Critical Perspective, dir. Maria Lirola (Cambridge: Cambridge Scholar Publishing, 2012); Andreas Mussolf, «Dehumanizing Metaphors in UK Immigrant Debates in Press and Online Media", Journal of Language Aggression and Conflict 3, no 1 (2015): 41-56; Arthur D. Santana, "Virtuous or Vitriolic», Journalism Practice 8, no 1 (2014): 18-33; Arthur D. Santana, «Incivility Dominates Online Comments on Immigration", Newspaper Research Journal 36, no 1 (2015): 92-107; Ian Rowe, "Civility 2.0: A Comparative Analysis of Incivility in Online Political Discussion ", Information, Communication \& Society 18, no 2 (2015): 121-38.

23 Clemence Due, " Aussie Humour” or Racism? Hey Hey It's Saturday and the Denial of Racism in Online Responses to News Media Articles", Platform 3, no 1 (2011): 36-53; Simon Goodman et Lottie Rowe, " "Maybe It Is Prejudice ... but It Is Nот Racism »: Negociating Racism in Discussion Forums about Gypsies », Discourse \& Society 25, no 1 (2013): 753-72; Gavan Titley, "The Debatability of Racism. Networked Participative Media and Postracialism», https:// raster.fi/2016/02/17/the-debatability-of-racism-networkedparticipative-media-and-postracialism/.

24 Par exemple, Burke et Goodman relèvent que les opposants aux réfugiés disent ne pas être racistes, ne pas parler de race, et se posent en victimes en dénonçant le tabou ou le politically correct. Burke et Goodman, " Bring Back Hitler's Gas Chambers" ".

25 Simon Weaver, "A Rhetorical Discourse Analysis of Online Anti-Muslim and Anti-Semitic Jokes », Ethnic and Racial Studies 36, no 3 (2013): 483-99; Tuuli Lädhesmäki et Tuija Saresma, «Reframing Gender Equality in Finnish Online Discussion on Immigration: Populist Articulations of Religious Minorities and Marginalized Sexualities", Nordic Journal of Feminist and Gender Research 22, no 4
(2014): 299-313; Imran Awan, «Islamophobia and Twitter: A Typology of Online Hate against Muslims on Social Media", Policy \& Internet 6, no 2 (2014): 133-50; Imran Awan, Islamophobia in Cyberspace: Hate Crime Go Viral (Farnham: Ashgate, 2016); Chris Allen, «Anti-Social Networking: Findings from a Pilot Study on Opposing Dudley Mosque Using Facebook as Both Site and Method for Research», SAGE Open, no Janvier-Mars (2014); Mattias Ekman, "Online Islamophobia and the Politics of Fear: Manufacturing the Green Scare», Ethnic and Racial Studies 38, no 11 (2015): 1986-2002; Steve Garner et Saher Selod, "The Racialization of Muslims: Empirical Studies of Islamophobia», Critical Sociology 41, no 1 (2015): 9-19; En-Chieh Chao, "The-Truth-about-Islam.Com: Ordinary Theories of Racism and Cyber Islamophobia ", Critical Sociology 41, no 1 (2015): 57-75; Chaudry, «\#Hashtagging Hate».; Andre Oboler, "The Normalisation of Islamophobia through Social Media», dans Islamophobia in Cyberspace, dir. Imran Awan (Milton Park: Routledge, 2016). Même lorsque les propos ouvertement racistes sont peu nombreux, des discours au langage codé peuvent donner lieu à une démarcation entre "Nous» nationaux blancs "normaux» et «Eux» musulmans menaçants.

26 Gavan Titley, «No Apologies for Cross-Posting: European Trans-Media Space and the Digital Circuitries of Racism», Crossings: Journal of Migration \& Culture 5, no 1 (2014): 41-55.

27 Le terme «immigrant» renvoie à toute personne résidant au Canada et étant né à l'étranger et inclus donc les réfugiés.

28 Andrea Rea, Sociologie de l'immigration (Paris: La Découverte, 2008).; Charles Taylor, Multiculturalisme. Différence et démocratie (Paris: Flammarion, 2009); Greg Noble, «The Discomfort of Strangers: Racism, Incivility and Ontological Security in a Relaxed and Comfortable Nation", Journal of intercultural studies 26, no 1-2 (2005): 107-120.

29 Hughey et Daniels, "Racist Comments at Online News Sites".

30 Teun A. Van Dijk, "Discourse and Racism», dans A Companion to Racial and Ethnic Studies, dir. David Theo Goldberg et John Solomos (Oxford: Wiley-Blackwell, 2002); «Racist Discourse, dans Encyclopedia of Race and Ethnic Studies, dir. Ellis Cashmore (Londres: Routledge, 2004); Ruth Wodak et al., The Discursive Construction of National Identity, 2 éd. (Edinbourg: Edinburgh University Press, 2009).

31 Robert Miles, Racism (Londres: Routledge, 1989); Balibar, "Racisme et nationalisme»; David Theo Goldberg, The Threat of Race: Reflections on Racial Neoliberalism (Malden: Wiley-Blackwell, 2009).

32 Notre utilisation du concept de patrouille des frontières suit celle de Sirma Bilge. Voir Sirma Bilge, «“... alors que nous, Québécois, nos femmes sont égales à nous et nous les aimons ainsi": la patrouille des frontières au nom de l'égalité de genre dans une "nation" en quête de souveraineté», Sociologie et sociétés 42, no 1 (2010): 197-226. 
33 Nous appliquons donc à la catégorie nation la perspective théorique selon laquelle les frontières «Nous» / «Eux» se construisent au sein de rapports sociaux matériellement et symboliquement inégaux soutenant et soutenus par des processus de différenciation et d'attribution catégorielle au sein desquels des marqueurs sont sélectionnés par les majoritaires pour distinguer l'Autre. Voir Danielle Juteau, Lethnicité et ses frontières (Montréal: Les Presses de l'Université de Montréal, 1999); Floya Anthias et Nira Yuval-Davis, Racialized Boundaries. Race, Nation, Gender, Colour and Class and the Anti-Racist Struggle (Londres: Routledge, 1992).

34 Stuart Hall, "The West and the Rest: Discourse and Power", dans Modernity. An Introduction to Modern Societies, dir. David Held, Don Hubert et Kenneth Thompson (Hoboken: Wiley-Blackwell, 1996), 225.

35 "Gramsci's Relevance for the Study of Race and Ethnicity", Journal of Communication Inquiry 10, no 2 (1986); Stuart Hall et Les Back, "In Conversation: At Home but Not at Home», Cultural Studies 23, no 4 (2009): 656-87; Stuart Hall et Doreen Massey, "Interpreting the Crisis », dans The Neoliberal Crisis, dir. Jonathan Rutherford et Sally Davison (Londres: Lawrence and Wishart, 2012).

36 Martha Augoustinos et Danielle Every, «The Language of "Race" and Prejudice: A Discourse of Denial, Reason, and Liberal-Practical Politics ", Journal of Language and Social Psychology 26, no 2 (2007): 123-41; Barnor Hesse, «SelfFulfilling Prophecy: The Post-Racial Horizon», South Atlantic Quaterly 110, no 1 (2011): 155-78; Steve Garner, A Moral Economy of Whiteness. Four Frames of Racializing Discourse (Londres: Routledge, 2016).

37 Teun A. Van Dijk, "Discourse and the Denial of Racism», Discourse \& Society 3, no 1 (1992): 87-118; Anna Triandafyllidou, Immigrants and National Identity in Europe (Londres: Routledge, 2001); Garner, Moral Economy of Whiteness.

38 L'islamophobie est ici entendue comme une forme spécifique de racisme. Elle ne renvoie pas à la critique de la religion musulmane, mais bien à la construction de la figure racialisée du musulman comme altérité menaçante prémoderne, agressive et culturellement incompatible avec l'Occident. S'il s'agit d'un phénomène global, il varie en fonction des contextes nationaux et commande un examen empirique. Voir Ramón Grosfoguel et Eric Mielants, «The Long-Durée Entanglement between Islamophobia and Racism in the Modern/Colonial Capitalist/Patriarchal World-System: An Introduction", Human Architechture: Journal of the Sociology of Self-Knowledge 5, no 1 (2006): 1-12; Abdellali Hajjat et Marwan Mohammed, Islamophobie: Comment les élites françaises fabriquent le "problème musulman» (Paris: La découverte, 2013).; Garner et Selod, «The Racialization of Muslims: Empirical Studies of Islamophobia»; Ghassan Hage, Is Racism an Environmental Threat? (Cambridge: Polity Press, 2017).

39 Philomena Essed, «Racisme et préférence pour l'identique: du clonage culturel dans la vie quotidienne», Actuel Marx, no 38 (2005): 103-18; Les Back et Shamser Sinha, "New Hierarchies of Belonging", European Journal of Cultural Studies 15, no 2 (2012): 139-54.

40 Alana Lentin et Gavan Titley, The Crises of Multiculturalism. Racism in a Neoliberal Age (Londres: Zed Books, 2011).

41 Back et Sinha, "New Hierarchies of Belonging», 140.

42 Hage parle en termes de «nationalisme paranoïaque». Voir Ghassan Hage, Against Paranoid Nationalism. Searching for Hope in a Shrinking Society (Londres: Merlin, 2003).

43 Voir notamment Ghada Mahrouse, " Reasonable Accommodation" in Québec: The Limits of Participation and Dialogue», Race \& Class 52, no 1 (2010): 85-96; Sirma Bilge, "Reading the Racial Subtext of the Québécois Accommodation Controversy: An Analytics of Racialized Governmentality", Politikon 40, no 1 (2013): 157-81; Daiva Stasiulis, "Worrier Nation: Quebec's Value Codes for Immigrants", Politikon 40, no 1 (2013): 183-209; Maryse Potvin, «The Reasonable Accommodations Crisis in Quebec: Racializing Rhetorical Devices in Media and Social Discourse", International Journal of Canadian Studies 50 (2014): 137-61; Leila Benhadjoudja, "Laïcité narrative et sécularonationalisme au Québec à l'épreuve de la race, du genre et de la sexualité », Sciences Religieuses 46, no 2 (2017): 272-91.

44 Le concept de majoritaire désigne le groupe ethnique dominant aux niveaux économique, politique et symbolique. À l'inverse, le statut de minoritaire résulte du processus de minorisation sociale et non pas du statut de minorité démographique. Voir Juteau, L’ethnicité et ses frontières.

45 Hage conceptualise les sociétés comme des mécanismes de distribution de l'espoir. Pour lui, l'opposition à l'accueil de réfugiés en Occident ne peut donc être analysée qu'en prenant en compte le peu d'espoir avec lequel les majorités sont laissées sous le néolibéralisme. Voir Hage, Against Paranoid Nationalism.

46 Ces débats sont une forme ritualisée de la dialectique de l'inclusion et de l'exclusion où est visible la hiérarchisation racialisée des «étrangers» en relation avec la construction des frontières de la nation. Pour Hage, les majoritaires sérigent en sujets gouvernementaux au sein de ces rituels. C'est en vertu de leur capital national supérieur et naturalisé qu'ils se voient comme les arbitres et gestionnaires légitimes au sein de leur espace national. Suivant la théorie bourdieusienne, Hage avance que les acteurs cherchent à accumuler du capital national afin de le transférer en appartenance nationale et donc dêtre reconnus comme membres légitimes par le groupe dominant. Il s'agit donc d'un type de capital symbolique propre à ce champ. Or, le capital national des minorités racialisées est précaire puisqu'elles doivent le performer en permanence, c'est-àdire prouver culturellement leur appartenance nationale. Une distinction est donc opérée entre celles et ceux pour qui la possession de capital national relève d'une disposition naturelle et les autres qui ont dû acquérir ce capital qui, du fait de sa qualité acquise, est de valeur inférieure et 
requiert dêtre constamment démontré. Les premiers sont, par essence, nationaux alors que les minorités racialisées doivent s'efforcer de faire reconnaître leur appartenance en agissant nationalement.

47 Ruth Frankenberg, White Women, Race Matters: The Social Construction of Whiteness (Minneapolis: University of Minnesota Press, 1993). La blanchité est aussi entendue comme un fantasme de dominance culturelle et de propriété qui découle de la colonisation. Sur la blanchité, voir Stuart Hall, «Culture, Community, Nation », Cultural Studies 7, no 3 (1993): 349-63; Aileen Moreton-Robinson, The White Possessive. Property, Power, and Indigenous Sovereignity (Minneapolis: University of Minneapolis Press, 2015); Barnor Hesse, "Racialized Modernity: An Analytics of White Mythologies", Ethnic and Racial Studies 30, no 4 (2007): 643-63; Garner, "Moral Economy of Whiteness»; Hage, Writings on Nationalism.

48 Par exemple, aux Pays-Bas, le déni du racisme systémique et quotidien saccompagne du déni de la blanchité comme attribut des «vrais» nationaux. Suivant la conceptualisation proposée par David Theo Goldberg, Essed et Trienekens considèrent la situation néerlandaise comme caractéristique de leuropéanisation raciale où les processus complexes d'altérisation au cœur de lévaluation du degré d'acquisition de l'appartenance aux yeux de la majorité dominante font appel à la nationalité, l'ethnicité, la race, la corporalité et la religion, et ce tout en subsumant la race sous le couvert de références culturelles et religieuses. Philomena Essed et Sandra Trieneckens, " "Who Wants to Feel White?" Race, Dutch Culture and Contested Identities", Ethnic and Racial Studies 31, no 1 (2008): 52-72.

49 Cette idée de collage renvoie au concept de «sticking» de Sara Ahmed. Sa théorie des économies affectives avance que, par la circulation des signes de peur, les émotions fonctionnent en collant ensemble des figures de façon telles que certaines mobilités sont associées à des signes de peur. Par exemple, à travers sa circulation, le mot terroriste colle à certains corps plus quà d'autres et se déplace dans d'autres termes comme "fondamentalisme», "Arabe» et «Islam». Voir Sara Ahmed, «Affective Economies», Social Text 22, no 2 (2004): 117-39.

50 Garner, Moral Economy of Whiteness, 142.

51 Cela se reflète d'ailleurs dans un rapport de distance à l'identité québécoise chez les jeunes «issus de l'immigration». Voir Maryse Potvin, «Blackness, haïtianité et québécitude: modalités de participation et d'appartenance chez la deuxième génération d'origine haïtienne au Québec», dans La ze génération issue de l'immigration: Une comparaison France-Québec, dir. Maryse Potvin, Paul Eid et Nancy Venel (Outremont: Athéna Éditions, 2007); Gina Lafortune et Fasal Kanouté, «Vécu identitaire délèves de 1ère et de zème génération d’origine haitienne", Revue de l'Université de Moncton 38, no 2 (2007): 33-71; Marie-Odile Magnan, Fahimeh Darchinian et Émilie Larouche, «Identifications et rapports entre majoritaires et minoritaires. Discours de jeunes issus de l'immigration", Diversité urbaine 17 (2017): 29-47.

52 Vron Ware, «Towards a Sociology of Resentment: A Debate on Class and Whiteness", Sociological Research Online 13, no 5 (2008); Philomena Essed et Isabel Hoving, «Innocence, Smug Ignorance, Resentment: An Introduction to Dutch Racism ", dans Dutch Racism, dir. Philomena Essed et Isabel Hoving (Amsterdam: Rodopi, 2014).

53 Ces médias ont été sélectionnés en fonction de leur importance au sein du paysage médiatique, mais aussi de façon à inclure des sources de différents formats journalistiques et orientations politiques.

54 Notons que la littérature fait état d'une relation entre le type d'opinion par rapport à l'immigration et la source d'information journalistique privilégiée, les lecteurs des tabloïds étant plus nombreux à exprimer des opinions hostiles. Voir John Sides et Jack Citrin, «European Opinion about Immigration: The Role of Identities, Interests and Information», Bristish Journal of Political Science 37, no 3 (2007): 477-504; Lauren McLaren et Mark Johnson, «Resources, Group Conflict and Symbols: Explaining AntiImmigration Hostility in Britain ", Political Studies 55, no 4 (2007): 709-32.

55 Cette catégorisation s'appuie sur le nom des commentateurs. La très forte majorité des commentateurs semblent s'exprimer avec leur nom véritable et non pas un pseudonyme. En cas de doute, la catégorisation pouvait aussi prendre appui sur la photo de profil jointe au commentaire et sur des indicateurs dans les propos soutenus, par exemple une référence aux ancêtres ou une opposition entre «Nous» et «les immigrants». Il s'agit évidemment donc d'une méthode faillible dépendante du type de données analysées. Une telle catégorisation repose aussi sur trois suppositions qui ne peuvent être vérifiées, soit que le commentaire est rédigé par une personne réelle et non un bot, que les informations comme le nom et la photo sont véridiques et que les noms interprétés comme signalant une identité ethnique canadienne-française signifient aussi que les personnes sont blanches/majoritaires.

56 Voir Teun A. Van Dijk, "Critical Discourse Analysis", dans Handbook of Discourse Analysis, dir. Deborah Schiffrin, Deborah Tannen et Heidi E. Hamilton (Oxford: Blackwell, 2001); Martin Reisigl et Ruth Wodak, «The Discourse-Historical Approach (DHA)», dans Methods of Critical Discourse Analysis, dir. Ruth Wodak et Michael Meyer (Londres: SAGE, 2009); Norman Fairclough, Jane Mulderrig et Ruth Wodak, "Critical Discourse Analysis", dans Discourse Studies. A Multidisciplinary Introduction, dir. Teun A. Van Dijk (Londres: SAGE, 2011).

57 Ruth Wodak, «Pragmatics and Critical Discourse Analysis: A Cross-Disciplinary Inquiry», Pragmatics \& Cognition 15, no 1 (2007): 203-25.

58 Voir David Beer et Roger Burrows, "Sociology and, of and in Web 2.0: Some Initial Considerations», Sociological Research Online 12, no 5 (2007); Majid KhosraviNik, 
«Critical Discourse Analysis, Power and New Media: Issues and Debates,» dans Why Discourse Matters: Negotiating Identity in the Mediatized World, dir. Yusuf Kalyango Jr. et Monika Weronika Kopytowska (New York: Peter Lang, 2014); Majid KhosraviNik et Johann W. Unger, «Critical Discourse Studies and Social Media: Power, Resistance and Critique in Changing Media Ecologies», dans Methods of Critical Discourse Studies, dir. Ruth Wodak et Michael Meyer (Londres: SAGE, 2016).

59 Les topoi sont des prémisses du discours servant de justification en connectant largument et la conclusion. Parmi les topoi prévalents dans les discours sur l'immigration, on retrouve l'utilité/inutilité, le danger/la menace, la responsabilité, le fardeau, léconomie, l'histoire, la culture, etc. Ruth Wodak, "The Semiotics of Racism. A Critical Discourse Analysis", dans Discourse, of Course: An Overview of Research in Discourse Studies, dir. Jan Renkema (Amsterdam: John Benjamins Publishing, 2009).

6o Teun A. Van Dikj, Ideology. A Multidisciplinary Approach. (Thousand Oaks: SAGE, 1998).

61 Hughey et Daniels, "Racist Comments at Online News Sites», 336 .

62 Dans ce contexte, non seulement le langage raciste explicite est publiquement dénoncé, mais la race est invalidée pour expliquer la distribution inégale des positions et privilèges sociaux. Voir Van Dijk, «Discourse and Racism »; Augoustinos et Every, "Language of "Race" and Prejudice»; Goldberg, Threat of Race; Simon Goodman, «Developing an Understanding of Race Talk», Social and Personality Psychology Compass 8, no 4 (2014): 147-55; Alana Lentin, «Racism in Public or Public Racism: Doing Anti-Racism in “Post-Racial» Times», Ethnic and Racial Studies (2015): 1-16.

63 Van Dijk, "Discourse and the Denial of Racism"; Halleh Ghorashi, "Racism and "the Ungrateful Other" in the Netherlands» dans, Dutch Racism, dir. Philomena Essed et Isabel Hoving (Amsterdam: Rodopi, 2014).

64 Hage, Writings on Nationalism.

65 Van Dijk, «Discourse and Racism», 157.

66 Sivamohan Valluvan, «Defining and Challenging the New Nationalism ", Progressive Review 23, no 4 (2017): 232-39.

67 Ruth Wodak, "“Us and Them": Inclusion and Exclusion - Discrimination via Discourse», dans Identity, Belonging and Migration, dir. Gerard Delanty, Ruth Wodak et Paul Jones (Liverpool: Liverpool University Press, 2008), 65.

68 Augoustinos et Every, "The Language of "Race" and Prejudice»; Goodman, «Developing an Understanding of Race Talk».

69 Le terme $B S$ vient de l'ancien nom de l'aide sociale au Québec, soit le bien-être social. Les prestataires de l'aide sociale forment une catégorie de la population faisant l'objet d'une forme importante de méfiance au Québec, une personne sur deux affirmant en avoir une opinion négative. Voir Pierre Noreau et al., "Droits de la personne et diversité» (Commission des droits de la personne et des droits de la jeunesse, 2015).
70 James P. Walsh, «Navigating Globalization: Immigration Policy in Canada and Australia, 1945-2007», Sociological Forum 23, no 4 (2008): 217-28; Walsh, "Quantifying Citizens: Neoliberal Restructuring and Immigrant Selection in Canada and Australia ", Citizenship Studies 15, no 6-7 (2011): 861-79; Harold Bauder, Immigration Dialectic: Imagining Community, Economy, and Nation (Toronto: University of Toronto Press, 2011); Forcier et Dufour, «Immigration, Neoconservatism and Neoliberalism»; Susan Barrass et John Shields, «Immigration in an Age of Austerity: Morality, the Welfare State and the Shaping of the Ideal Migrant», dans Austerity: The Lived Experience, dir. Bryan M. Evans et Stephen McBride (Toronto: University of Toronto Press, 2017).

$71 \mathrm{Au}$ Canada, l'appui aux niveaux d'immigration élevés est indissociable du système de sélection et de la croyance partagée voulant que cette immigration ait un impact positif sur l'économie nationale. Voir Jeffrey G. Reitz, «Proimmigration Canada. Social and Economic Roots of Popular Views», IRPP Study, no 20 (2011).; Irene Bloemraad, «Understanding "Canadian Exceptionalism" in Immigration and Pluralism Policy", dans Migration Policy Institute (Washington: Transatlantic Council on Immigration, 2012). Le support des Canadiens pour l'immigration en provenance des "pays pauvres » est comparable à celles des Européens. Voir Michael J. Donnelly, "Canadian Exceptionalism: Are We Good or Are We Lucky?» (Montreal: McGill Institute for the Study of Canada, 2017).

72 Ruth Wodak, The Politics of Fear: What Right-Wing Populist Discourses Mean (Thousand Oaks: SAGE, 2015).

73 Ware, "Towards a Sociology of Resentment»; Steve Fenton, «Resentment, Class and Social Sentiments about the Nation: The ethnic Majority in England», Ethnicities 12, no 4 (2012): 465-83.

74 Garner, «Moral Economy of Whiteness ».

75 Michael Billig, Banal Nationalism (Londres: Sage, 1995); Wodak, " Us and Them" »; Wodak et al., Discursive Construction of National Identity.

76 Reisigl et Wodak, «The Discourse-Historical Approach (DHA)».

77 Sara Ahmed, The Cultural Politics of Emotion (Edinbourg: Edinburgh University Press, 2004).

78 Ruth Wodak, "The "Establishment", the "Élites", and the "People". Who's Who?», Journal of Language and Politics 16, no 4 (2017): 551-65.

79 Comme le note bien Hage, les pratiques nationalistes présupposent premièrement l'image d'un espace national, deuxièmement, l'image des nationalistes comme maittres de cet espace et, troisièmement, l'image d'Autres ethno-raciaux comme simples objets au sein de ce territoire. Larticulation entre spatialité, entitlement et solidarité sélective transparaît notamment dans le recours à des termes appuyant la logique d'appartenance gouvernementale où les majoritaires positionnent les minorités racialisées au sein de la hiérarchie des appartenances. 
80 Sara Ahmed, Strange Encounters. Embodied Others in PostColoniality (Londres: Routledge, 200o); Hall et Back, «In Conversation".

81 Cela rejoint l'idée de conceptualiser le welfare chauvinism en termes de continuum. Il renvoie de façon sommaire au ciblage des immigrants et des minorités au sein de la restructuration néolibérale de l'État et à la question à savoir si tous devraient avoir un accès égal aux ressources de l'État que les natifs. Nous ne réservons donc pas l'utilisation du terme pour les discours d'extrême-droite qui revendiquent à un accès exclusif pour les majoritaires blancs. Voir Tim Reeskens et Wim Van Oorshot, «Disentangling the "New Liberal Dilemma": On the Relations between General Welfare Distribution Preferences and Welfare Chauvinism ", International Journal of Comparative Sociology 53, no 2 (2012): 120-39; Martin Bak Jørgensen et Trine Lund Thomsen, «Deservingness in the Danish Context: Welfare Chauvinism in Times of Crisis", Critical Social Policy 36, no 3 (2016): 330-51.

82 Ghassan Hage, "On Worrying: The Lost Art of the WellAdministered National Cuddle», Borderlands 2, no 1 (2003); Gavan Titley, "Exclusion through Openness? A Tentative Anatomy of the Ritual of "Migration Debates" ", Studies across Disciplines in the Humanities and Social Sciences 11 (2012): 46-69.

83 De tels discours concevant la nation comme une grande famille dont les membres doivent être protégés et défendus vis-à-vis de menaces externes montre bien la relation entre les imaginaires nationalistes et racistes. Voir Stuart Hall, «The Question of Cultural Identity», dans Modernity: An Introduction to Modern Societies, dir. Stuart Hall et al. (Oxford: Blackwell, 1996); Balibar, «La forme nation».

84 Garner, Moral Economy of Whiteness, 82.

85 Voir Allison Harell, Stuart Soroka et Kiera Ladner, «Public Opinion, Prejudice and the Racialization of Welfare in Canada», Ethnic and Racial Studies 37, no 14 (2014): 258097; Allison Harell, Stuart Soroka et S. Iyengar, «Race, Prejudice and Attitudes toward Redistribution: A Comparative Experimental Approach", European Journal of Political Research 55, no 4 (2016): 723-44; Stuart Soroka, Matthew Wright, Richard Johnston, Jack Citrin, Keith Banting et Will Kymlicka, «Ethnoreligious Identity, Immigration, and Redistribution », Journal of Experimental Political Science 4, no 3 (2017): 173-82.

86 Paul M. Sniderman, Louk Hagendoorn et Markus Prior, «Predisposing Factors and Situational Triggers: Exclusionary Reactions to Immigrant Minorities", American Political Science Review 98, no 1 (2004): 35-49; Edward Anthony Koning, «Selective Solidarity: The Politics of Immigrants' Social Rights in Western Welfare States » (Queen's University, 2013); Will Kymlicka, «Solidarity in Diverse Societies: Beyond Neoliberal Multiculturalism and Welfare Chauvinism », Comparative Migration Studies 3, no 17 (2015): 1-19; Frederik Hjorth, «Who Benefits? Welfare Chauvinism and
National Stereotypes", European Union Politics 17, no 1 (2016): 3-24.

87 Lentin et Titley, Crises of Multiculturalism; Aurelien Mondon et Aaron Winter, "Articulations of Islamophobia: From the Extreme to the Mainstream?», Ethnic and Racial Studies 40, no 13 (2017): 2151-79.

88 Philomena Essed et Kwame Nimako, «Designs and (Co) Incidents: Cultures of Scholarship and Public Policy on Immigrants/Minorities in the Netherlands", International Journal of Comparative Sociology 47, no 3-4 (2006): 281-312; Gilbert Caluya, «Domestic Belongings: Intimate Security and the Racial Politics of Scale», Emotion, Space and Society 4, no 4 (2011): 203-10; Ghorashi, "Racism and "The Ungrateful Other" in the Netherlands ».

89 Ahmed, Strange Encounters.

90 Edward Saï, "Orientalism Reconsidered», Cultural Critique 1 (1985): 89-107.

91 Jesse A. Steinfeldt et al., «Racism in the Electronic Age: Role of Online Forums in Expressing Racial Attitudes about American Indians ", Cultural Diversity and Ethnic Minority Psychology 16, no 3 (2010): 362-71; Due, «"Aussie Humour" or Racism»; Burke et Goodman, " "Bring Back Hitler's Gas Chambers" »; Hughey et Daniels, «Racist Comments at Online News Sites»; Goodman et Rowe, " "Maybe It Is Prejudice" »; Titley, "No Apologies for Cross-Posting»; Garner, Moral Economy of Whiteness.

92 Voir George Morgan et Scott Poynting, dir., Global Islamophobia: Muslims and Moral Panic in the West (Farnham: Ashgate, 2012).

93 Sherene Razack, Casting Out the Eviction of Muslims from Western Law and Politics (Toronto: University of Toronto Press, 2008); Goldberg, Threat of Race.

94 Voir Jill Walker Rettberg et Radhika Gajjala, «Terrorists or Cowards: Negative Portrayals of Male Syrian Refugees in Social Media», Feminist Media Studies 16, no 1 (2016): 178-81.

95 Notons que le gouvernement canadien a annoncé en novembre 2015 que, pour des raisons de sécurité, les hommes seuls ne seraient pas admis. Les femmes et les familles étaient alors présentées comme étant des réfugiés sécuritaires, ce qui contribuait à cadre les hommes célibataires comme étant de potentiels faux-réfugiés et terroristes. Peu avant cette annonce, le maire de Québec Régis Labeaume avait affirmé être disposé à accueillir des orphelins et des familles, mais pas des "gars de 20 ans frustrés». Dans les commentaires, une telle position est souvent présentée comme étant raisonnable, appartenant au «gros bon sens».

96 Ashley Bradimore et Harald Bauder, «Mystery Ships and Risky Boat People: Tamil Refugee Migration in the Newsprint Media", Canadian Journal of Communication 36, no 4 (2011): 637-60; Liette Gilbert, «The Discursive Production of a Mexican Refugee Crisis in Canadian Media and Policy ", Journal of Ethnic and Migration Studies 39, no 5 (2013): 827, 843; Dagmar Soennecken, «Shifting Up and Back. The 
European Turn in Canadian Refugee Policy", Comparative Migration Studies 2, no 1 (2014): 101-22; David Moffette et Shaira Vadasaria, «Uninhibited Violence: Race and the Securitization of Immigration », Critical Studies on Security 4, no 3 (2016): 391-405.

97 Sur les effets de la Loi visant à protéger le système d'immigration du Canada, voir Idil Atak, Graham Hudson et Delphine Nakache, «The Securitisation of Canada's Refugee System: Reviewing the Unintended Consequences of the 2012 Reform ", Refugee Survey Quaterly 37, no 1 (2018): 1-24.

98 D’ailleurs, plusieurs justifient l'exclusion des réfugiés en arguant que la solution devrait passer par une intervention militaire en Syrie. Ce pays et le Moyen-Orient plus largement sont alors souvent perçus comme étant des territoires de guerres de religions menées par les musulmans. Le portrait est alors celui d'une région dominée par des mœurs barbares. Suivant cela, une différenciation est opérée entre un Occident pacifique et moderne d'un côté et un Orient guerrier et pré-moderne de l'autre. En ce sens, si l'Occident a un rôle à jouer dans ce conflit, ce serait celui d' «aider» les Syriens «chez eux» afin qu'ils puissent ultimement se gouverner et reprendre le contrôle de «leur» pays. Largument est alors que, non seulement les réfugiés syriens représentent une menace sécuritaire, mais aussi que l'Occident a le fardeau de devoir aider ces Autres dans leur territoire d'appartenance.

99 William Walters, "Secure Borders, Safe Haven, Domopolitics », Citizenship Studies 8, no 3 (2004): 237-6o.

100 Moffette et Vadasaria, "Uninhibited Violence»; William Walters, «Migration and Security, " dans The Handbook of
New Security Studies, dir. Peter J. Burgess (Londres: Routledge, 2010).

101 Alexandra Dobrowolsky, "(In)Security and Citizenship: Security, Im/migration and Shrinking Citizenship Regimes", Theoretical Inquiries in Law 8, no 2 (2007): 629, 719; Michael Humphrey, «Migration, Security and Insecurity ", Journal of Intercultural Studies 34, no 2 (2013): 178-95.

102 Sara Ahmed, "Affective Economies", Social Text 22, no 2 (2004): 117-39.

103 À cet effet, voir Garner, Moral Economy of Whiteness; Michael Skey, «Boundaries and Belonging: Dominant Ethnicity and the Place of the Nation in a Globalizing World", dans Nationalism, Ethnicity and Boundaries. Conceptualising and Understanding Identity through Boundary Approaches, dir. Jennifer Jackson et Lina Molokotos-Liederman (Londres: Routledge, 2015); Essed et Trieneckens, " "Who Wants to Feel White?"».

104 Wodak, "The "Establishment", the "Élites", and the "People" ".

105 Notons que cette proposition va à l'encontre du droit canadien de l'immigration. D’une part, la catégorie des réfugiés est sous la responsabilité de l'État fédéral. D’autre part, la résidence permanente est délivrée par le Canada et non pas le Québec. Le statut de ces immigrants durant leurs trois premières années de résidence est donc pour le moins imprécis.

Mathieu Forcier est candidat au doctorat en sociologie à l'Université de Montréal. Il peut être contacté au mathieu .forcier@umontreal.ca 